\title{
Article
}

\section{International Multicentre Study of Candida auris Infections}

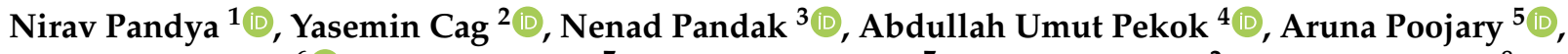 \\ Folusakin Ayoade ${ }^{6}{ }^{\circ}$, Teresa Fasciana ${ }^{7}$, Anna Giammanco ${ }^{7}$, Hulya Caskurlu ${ }^{2}$, Dhanji P. Rajani ${ }^{8}$, \\ Yogesh Kumar Gupta ${ }^{9}$, Ilker Inanc Balkan ${ }^{10}$ (D) Ejaz Ahmed Khan ${ }^{11}\left(\mathbb{D}\right.$ and Hakan Erdem ${ }^{12, *(1)}$
}

1 Consultant Microbiologist \& Infection Preventionist, Bhailal Amin General Hospital, Vadodara 390003, India; drniravpandya@gmail.com

2 Department of Infectious Diseases \& Clinical Microbiology, Istanbul Medeniyet University, Faculty of Medicine, Istanbul 34734, Turkey; dryasemincag@gmail.com (Y.C.); hcaskurlu@hotmail.com (H.C.)

3 The Royal Hospital, Muscat 111, Oman; npandak@gmail.com

4 VM Medical Park, Pendik Hospital, Istanbul 34899, Turkey; umutpekok@yahoo.com.tr

5 Breach Candy Hospital Trust, Mumbai 400026, India; arunapoojary@gmail.com

6 Division of Infectious Diseases, University of Miami, Miller School of Medicine, Miami, FL 33136, USA; fxa375@med.miami.edu

7 Department of Health Promotion, Mother and Child Care, Internal Medicine and Medical Specialities, University of Palermo, 90133 Palermo, Italy; teresa.fasciana@virgilio.it (T.F.); anna.giammanco@unipa.it (A.G.)

8 Microcare Laboratory \& TRC, Surat 395003, India; microcaresurat@gmail.com

9 Fortis Escorts Hospital, Jaipur 302017, India; dr.yogeshmicro@gmail.com

10 Cerrahpaşa Medical School, Istanbul University, Cerrahpaşa 34096, Turkey; ilkerinancbalkan@hotmail.com

11 Shifa International Hospital, Shifa Tameer e Millat University, Islamabad 44000, Pakistan; ejazkhan99@hotmail.com

check for updates

Citation: Pandya, N.; Cag, Y.; Pandak, N.; Pekok, A.U.; Poojary, A.; Ayoade, F.; Fasciana, T.; Giammanco, A.; Caskurlu, H.; Rajani, D.P.; et al. International Multicentre Study of Candida auris Infections. J. Fungi 2021, 7, 878. https://doi.org/10.3390/ jof7100878

Academic Editor: Sofia Costa-de-Oliveira

Received: 18 September 2021 Accepted: 12 October 2021 Published: 19 October 2021

Publisher's Note: MDPI stays neutral with regard to jurisdictional claims in published maps and institutional affiliations.

Copyright: (c) 2021 by the authors. Licensee MDPI, Basel, Switzerland. This article is an open access article distributed under the terms and conditions of the Creative Commons Attribution (CC BY) license (https:/ / creativecommons.org/licenses/by/ $4.0 /)$.
12 Department of Infectious Diseases, Bahrain Oncology Center, King Hamad University Hospital, Busaiteen 24343, Bahrain

* Correspondence: erdemhakan@gmail.com

\begin{abstract}
Background: Candida auris has emerged globally as a multi-drug resistant yeast and is commonly associated with nosocomial outbreaks in ICUs. Methods: We conducted a retrospective observational multicentre study to determine the epidemiology of $C$. auris infections, its management strategies, patient outcomes, and infection prevention and control practices across 10 centres from five countries. Results: Significant risk factors for C. auris infection include the age group of 61-70 years $(39 \%)$, recent history of ICU admission (63\%), diabetes (63\%), renal failure $(52 \%)$, presence of CVC $(91 \%)$ and previous history of antibiotic treatment (96\%). C. auris was commonly isolated from blood $(76 \%)$. Echinocandins were the most sensitive drugs. Most common antifungals used for treatment were caspofungin (40\%), anidulafungin (28\%) and micafungin (15\%). The median duration of treatment was 20 days. Source removal was conductedin $74 \%$ patients. All-cause crude mortality rate after 30 days was $37 \%$. Antifungal therapy was associated with a reduction in mortality (OR:0.27) and so was source removal (OR:0.74). Contact isolation precautions were followed in $87 \%$ patients. Conclusions: $C$. auris infection carries a high risk for associated mortality. The organism is mainly resistant to most azoles and even amphotericin-B. Targeted antifungal therapy, mainly an echinocandin, and source control are the prominent therapeutic approaches.
\end{abstract}

Keywords: Candida; C. auris; fungi; outbreak; nosocomial; resistance

\section{Introduction}

Candida auris is an emerging multidrug-resistant yeast that is spreading rapidly worldwide [1]. Genetic analyses show that five genetically different clades of C. auris emerged simultaneously from diverse geographical sites of different continents [2]. Infections caused by Candida species including $C$. auris are commonly detected in patients with prolonged hospitalization, especially in intensive care units (ICUs) [2,3]. It causes diseases ranging from superficial skin infections to invasive bloodstream infections (BSI) with high 
mortality rates $(30 \%$ to $60 \%)$ [4]. In addition, the difficulty faced in the identification, incorrect use of antifungal drugs, and treatment failure are associated with a high mortality rate [5]. C. auris is associated with nosocomial outbreaks, in intensive care settings, and transmission-despite the implementation of enhanced infection prevention and control (IPC) measures-is a particular concern [6,7]. Some strains of the organism are resistant to the three major classes of antifungals, severely limiting treatment options [8]. Furthermore, the relationship between minimal inhibitory concentration (MIC) values and clinical outcomes is still not completely assumed, resulting in a lack of consensus on the susceptibility breakpoints for $C$. auris [9].

The aims of this multicentre study are to analyse the epidemiology of C. auris infections, to study prevalence of various potential risk factors, its diagnosis and management strategies, patient outcomes and infection prevention and control (IPC) practices in hospitals across countries.

\section{Materials and Methods}

This was a retrospective multicentre study through ID-IRI platform (Infectious Disease International Research Initiative). Ethical approval was obtained from the local ethics committee on 27 May 2019 (Approval code: 2019-39).

Data Collection: All patients with C. auris hospitalized in participant centres were included in the study and their data were submitted. An online questionnaire was prepared via Google Forms for each enrolled patient. In each centre, data were submitted by the researcher who is committed as per institutional agreement to collaborate in the study. The ten participating centres (Bhailal Amin General Hospital, Vadodara, India; The Royal Hospital, Muscat, Oman; Pendik Hospital, Bahcesehir University, Istanbul, Turkey; Breach Candy Hospital Trust Mumbai, India; University of Miami Hospital, Miami, Florida, USA; Surat Gastroenterology Hospital, Surat, India; Krishna Super specialty Hospital, Surat, India; Fortis Escorts Hospital Jaipur, India; Istanbul University-Cerrahpaşa, Cerrahpaşa Medical School, Turkey; Shifa International Hospital, Islamabad, Pakistan) submitted the data of $C$. auris isolates recovered from all clinical specimens collected between 1 January 2019 and 31 December 2019 through Google Forms. The form included parameters of demographics, risk factors, laboratory testing, treatment, outcome and infection control practices(Annexure-1).

Inclusion/Exclusion criteria:

1. When multiple specimens of a patient showed growth of $C$. auris, only the specimen which was identified as source of infection was considered.

2. When C. auris was isolated from multiple cultures of a single patient, only one isolate, preferably from a sterile body site, was included in the study.

Identification: In seven centres, identification of the fungus was performed with Vitek-2 according to the manufacturer's instructions, using the latest software update (version 8.01), while in three centres, it was performed by matrix-assisted laser desorption ionization-time of flight mass spectrometry (MALDI-TOF) (Vitek mass spectrometer [MS], clinical knowledge database v3.2; bioMérieux).

Determining resistance: The antifungal susceptibility testing (AFST) was determined using two different methods: the broth microdilution method (BMD) (used by 2 centres) and Vitek 2 Compact system (used by 8 centres).

1. Clinical and Laboratory Standards Institute broth microdilution method (CLSI-BMD), following the M27-A3 guidelines. The antifungals tested were amphotericin B, fluconazole, voriconazole, caspofungin, micafungin, and anidulafungin.

2. Vitek-2 Compact system using an AST-YS07 or AST-YS08 card, which tests the MIC of the six antifungals (Amphotericin B, Voriconazole, Fluconazole, Caspofungin, and micafungin) $[10,11]$.

All the $C$. auris isolates were tested as per the manufacturer's instructions. There are no established CLSI/EUCAST breakpoints for C. auris. However, the CDC has provided 
tentative breakpoints and guidance for $C$. auris MIC interpretation, based on information collected for Candida spp. and numerous expert opinions [12,13]. These tentative breakpoints were used for interpretation of sensitivity results for micafungin, anidulafungin, caspofungin, amphotericin B and fluconazole. The MIC distribution of Candida auris isolates from various centres was also studied.

Outcome analysis was performed as 30 days outcome and defined as:

a. Resolution of infection and discharge of the patient;

b. Infection not resolved but patient was discharged;

c. Infection not resolved and patient was still hospitalized;

d. Patient transferred to other hospital;

e. Discharge against medical advice;

f. Death of the patient.

Infection control practices such as the screening of patients and their close healthcare contacts for multisite colonization of $C$. auris, appropriate transmission-based precautions and environmental disinfection were performed and analysed as per CDC recommendations [11]. Statistical analysis with calculation of percentage and odds ratio (OR) was performed using Microsoft Office Excel $2007^{\circledR}$ software (Redmond, WA, USA).

\section{Results}

A total of ten institutions from five countries participated in the study and submitted data for 54 patients. Data were analysed for risk factors, microbiology, treatment, patient outcomes and infection control practices. The most common age group was 61-70 (39\%) years, followed by $71-80(20 \%)$ years, $51-60(17 \%)$ years and $81-91(11 \%)$ years. Only $6 \%$ cases belonged to the $<40$ years of age group. The median age was 64.5 years. A total of 25 patients $(46 \%)$ were females. A total of 37 patients $(69 \%)$ were in the intensive care unit (ICU) and 17 were (32\%) in the wards. A total of 40 patients $(74 \%)$ had previous hospitalization history within the last 90 days, of which 34 patients $(63 \%)$ had a recent history of ICU admission.

\subsection{Risk Factors}

Comorbid conditions: The most common risk factors were diabetes ( $n=34,63 \%$ patients), followed by renal failure ( $n=28,52 \%$ patients), chronic obstructive pulmonary disease (COPD) or chronic pulmonary illness ( $n=21,39 \%$ patients) and congestive heart failure $(n=16,30 \%)$. A total of 14 patients $(26 \%)$ had one or other type of immunocompromised condition such asunderlying malignancy, HIV, severe combined immunodeficiency (SCID), liver transplant, non-Hodgkin lymphoma (NHL), or systemic lupus erythematosus (SLE).

Invasive Devices/procedures: $91 \%(n=49)$ of patients had a central venous catheter, $80 \%(n=43)$ had a urinary catheter, $69 \%(n=37)$ were on invasive ventilation, $37 \%(n=20)$ were on haemodialysis, and $24 \%(n=13)$ had a drain.

Therapeutic risk factors: The most common potential therapeutic risk factor for $C$. auris acquisition was a recent history of antibiotic treatment $(n=52,96 \%)$. Other probable risk factors were corticosteroid therapy $(n=19,35 \%)$, recent history of antifungal therapy $(n=15,28 \%)$, total parenteral nutrition $(n=12,22 \%)$, chemotherapy $(n=4,7 \%)$, and immunomodulatory therapy $(n=2,4 \%)$. Among 15 patients with a history of recent antifungal therapy, duration of antifungal exposure was more than 7 days in $73 \%(n=11)$ of patients. The most common antifungals used were echinocandins $(n=8,53 \%)$ and azoles $(n=6,40 \%)$. Only one patient $(7 \%)$ had a history of amphotericin-B therapy

ICU Stay: Out of 54 patients, 35 patients $(65 \%)$ had a hospital stay of more than one month. A total of 23 patients ( $43 \%$ ) had a stay of $1-2$ months, while 12 patients $(22 \%)$ had a stay of more than 3 months. In total, 39 out of 54 patients had an ICU stay $(72 \%)$, while the remaining 15 patients did not have any ICU stay. Of these 39 ICU patients, 25 patients $(90 \%)$ had an ICU stay of more than 1 week. 


\subsection{Microbiological Diagnosis}

Analysis of culture and sensitivity report of 54 C. auris isolates was performed. C. auris was most commonly isolated from blood $(n=41,76 \%)$. Other samples were skin and soft tissue $(n=6,11 \%)$, respiratory tract $(n=5,9 \%)$ and urine $(n=2,4 \%)$. Overall susceptibility to antifungal drugs was as shown in Figure 1. In this study, echinocandins were the most sensitive drugs followed by amphotericin-B and azoles.

\section{\%Susceptible - Antifungal drugs}

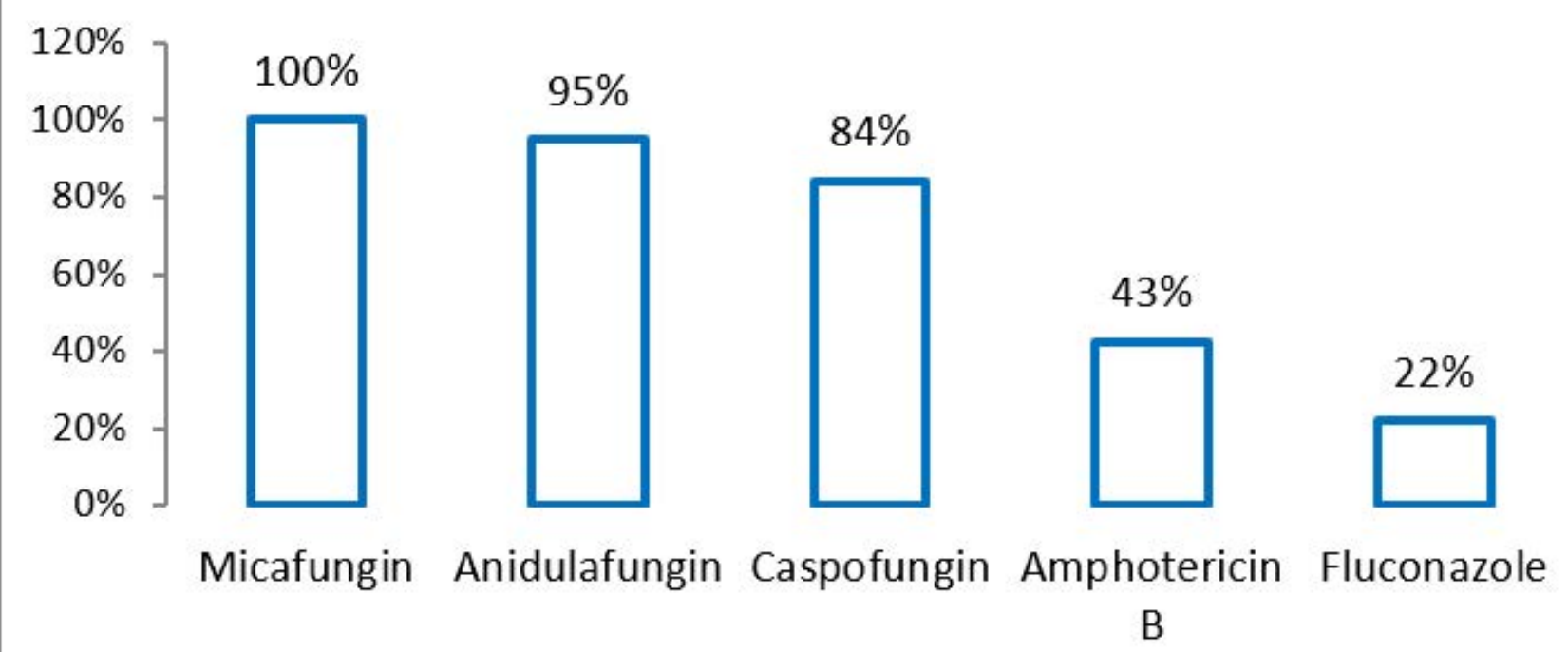

Figure 1. Susceptibility (\%) of antifungal drugs in Candida auris.

The minimum inhibitory concentration (MIC) data for C. auris isolates was provided by all the centres except one. MIC distribution of $C$. auris against eight antifungals is detailed in Table 1.

Table 1. MIC distribution of C. auris isolates.

\begin{tabular}{|c|c|c|c|c|c|c|c|c|c|c|c|c|c|c|c|c|c|c|}
\hline & $\leq 0.06$ & 0.12 & 0.25 & 0.5 & 1 & 2 & 4 & 8 & 16 & 32 & 64 & 128 & $\geq 256$ & Total $(n)$ & MIC Range & $\mathrm{GM}^{\mathrm{a}}$ & MIC50 ${ }^{b}$ & $\operatorname{MIC} 90^{c}$ \\
\hline Fluconazole & & & & & & & & 8 & 4 & $\underline{11 \mathrm{~d}}$ & 1 & & 6 & 30 & $8-\geq 256$ & 4.62 & 32 & $\geq 256$ \\
\hline Voriconazole & & 2 & 1 & 1 & $\underline{12}$ & 2 & 7 & 5 & & & & & & 30 & $\leq 0.12-\geq 8$ & 2.89 & 1 & $\geq 8$ \\
\hline Amphotericin B & & & & 6 & $\underline{10}$ & 4 & 2 & 1 & 7 & & & & & 30 & $0.5-\geq 16$ & 3.87 & 1 & $\geq 16$ \\
\hline Caspofungin & & 2 & $\underline{18}$ & 2 & $\overline{4}$ & 3 & 4 & & & & & & & 33 & $0.12-4$ & 3.89 & 0.25 & 4 \\
\hline Micafungin & 6 & $\underline{14}$ & & 2 & 6 & 4 & & & & & & & & 32 & $\leq 0.06-2$ & 5.26 & 0.12 & 2 \\
\hline Anidulafungin & 4 & $\overline{2}$ & 2 & 2 & $\underline{4}$ & 3 & 2 & & & & & & & 19 & $\leq 0.06-4$ & 2.58 & 0.5 & 4 \\
\hline Posaconazole & 3 & & 1 & & & & $\underline{9}$ & & & & & & & 13 & $0.06-4$ & 3.00 & 4 & 4 \\
\hline 5-Flucytosine & & & & & 3 & & & & 4 & 1 & $\underline{8}$ & & & 16 & $\leq 1-\geq 64$ & 3.13 & 32 & $\geq 64$ \\
\hline
\end{tabular}

${ }^{a}$ GM, geometric mean MICs. ${ }^{b}$ MIC50, MIC at which $50 \%$ of test isolates were inhibited. ${ }^{c}$ MIC 90 , MIC at which $90 \%$ of test isolates were inhibited. ${ }^{\mathrm{d}}$ Modal MICs (most repeated MIC value) are indicated with underlined numbers.

The CDC has not provided MIC breakpoints for voriconazole, posaconazole and 5-flucytosine against $C$. auris in its guideline document (12). Therefore, interpretation was not performed for these antifungals. However, as shown in Table 1, MIC distribution of these antifungals was determined. Notably, modal MIC of voriconazole was significantly lower $(1 \mathrm{mg} / \mathrm{L})$ than that of fluconazole $(32 \mathrm{mg} / \mathrm{L})$. Among echinocandins, modal MICs of micafungin, caspofungin and anidulafungin were $0.125,0.25$ and $1 \mathrm{mg} / \mathrm{L}$, respectively. MIC50 and modal MICs for all the antifungals were the same except for anidulafungin. MIC50 and modal MICs for anidulafungin were $0.5 \mathrm{mg} / \mathrm{L}$ and $1 \mathrm{mg} / \mathrm{L}$, respectively. MIC distribution of anidulafungin also showed an additional peak at MICs $\leq 0.06 \mathrm{mg} / \mathrm{L}$.

The duration from admission to first positive culture for C. auris was also analysed (Table 2). The median time from admission to diagnosis (positive culture) was 20 days. 
A total of 26 patients (48\%) also had concurrent growth of bacterial pathogens. Out of 26 patients, 12 patients (48\%) had growth of bacterial pathogens in their blood, $6(24 \%)$ in urine, $4(16 \%)$ in respiratory tissue, and $3(12 \%)$ in skin-soft tissue samples. Bacterial pathogens isolated from these patients are predominantly gram-negative organisms such as Klebsiella pneumoniae, Escherichia coli and Pseudomonas aeruginosa, as listed in Table 3. Repeat culture for C. auris was performed in 35 patients $(65 \%)$. Out of 35 , only $7(20 \%)$ were subsequently positive for $C$. auris, while $28(80 \%)$ turned out negative indicating microbiological clearance. Out of seven repeat positive patients, six were from blood and their repeat samples were taken within 7 days of first positive culture. In one patient repeat culture from post-CABG (coronary artery bypass grafting), chest wound was positive even after 63 days of the first positive culture, indicating wound colonization/chronic infection.

Table 2. Time from admission to positive culture.

\begin{tabular}{ccc}
\hline No. of Days & Patient No. & Patient \% \\
\hline$\leq 2$ days & 5 & $9 \%$ \\
$3-7$ days & 8 & $15 \%$ \\
$8-14$ days & 8 & $15 \%$ \\
15-30 days & 17 & $31 \%$ \\
$>1$ month & 16 & $30 \%$ \\
\hline
\end{tabular}

Table 3. Bacterial pathogens isolated from C. auris patients.

\begin{tabular}{ccc}
\hline Organism & No. of Organism & \% of Organisms \\
\hline Klebsiella pneumoniae & 7 & $24 \%$ \\
Escherichia coli & 6 & $21 \%$ \\
Pseudomonas aeruginosa & 5 & $17 \%$ \\
Staphylococcus aureus & 3 & $10 \%$ \\
Acinetobacter baumanii & 2 & $7 \%$ \\
Burkholderia cepacia & 2 & $7 \%$ \\
Stenotrophomonas maltophilia & 1 & $3 \%$ \\
Acinetobacter calcoaceticus & 1 & $3 \%$ \\
Enterococcus faecalis & 1 & $3 \%$ \\
Klebsiella ozaenae & 1 & $3 \%$ \\
Total & 29 & \\
\hline
\end{tabular}

\subsection{Therapeutic Issues}

Out of total 54 patients, 47 were treated with antifungal therapy, while 7 patients did not receive any antifungals. Out of these seven, fourpatients expired or discharged before availability of reports and threepatients were managed only with source control viz. central venous catheter (CVC) removal in twopatients and abscess drainage in 1 patient.

Drug of choice: In the 47 patients treated with antifungals, drug of choice was echinocandins in 39 patients (83\%) (caspofungin, $n=19,40 \%$; anidulafungin, $n=13$, $28 \%$; micafungin, $n=7,15 \%)$. Five patients $(11 \%)$ received fluconazole and one patient $(2 \%)$ received voriconazole. Besides, one patient received liposomal amphotericin B, while the other received combination of caspofungin and liposomal amphotericin-B.

Treatment as per susceptibility testing: Out of 47 patients receiving antifungals, 30 patients $(64 \%)$ were given treatment as per AFST report. Nine patients $(19 \%)$ received antifungal (anidulafungin) as per sensitivity result of surrogate echinocandins (caspofungin). Eight patients (17\%) received antifungals (seven echinocandins and one amphotericin-B) without AFST report, but based on identification of $C$. auris.

Dosing: 45 out of 47 patients received standard dosage of antifungals, as per CDC recommendations for C. auris [14]. Dose adjustment was conducted for Caspofungin in two patients with renal failure.

Duration of treatment: Out of 47 patients treated with antifungals, 25 patients $(53 \%)$ received more than 14 days of antifungals. $10(21 \%), 12(26 \%), 17(36 \%), 4(9 \%)$ and $4(9 \%)$ 
patients received $<1$ week, 1-2 weeks, 2-3 weeks, 3-4 weeks and $>4$ weeks of antifungal therapy, respectively. Out of 47 patients treated with antifungals, 13 patients $(28 \%)$ died, 2 patients (4\%) were discharged, and 2 patients $(4 \%)$ were transferred to other hospital. On the whole, 24 patients expired, and the median duration of antifungal treatment in remaining 30 survivors was 20 days.

Reasons for stopping antifungals: Out of 47 patients who received antifungals, a total of 28 patients $(60 \%)$ had microbiological clearance as evidenced by negative repeat culture. Reasons for stopping of antifungal treatment were microbiological clearance (51\%, $n=24$ patients), death $(28 \%, n=13$ patients), improved patient condition $(9 \%, n=4)$, completion of treatment duration $(4 \%, n=2)$, discharge $(4 \%, n=2)$ and transferred to other hospital $(4 \%, n=2)$.

Source removal: Source removal was done in 40 out of 54 patients (74\%). The most common approach was CVC removal within $24 \mathrm{~h}$ of positive culture in patients with Candidemia. Other approaches such as wound debridement and prosthetic material removal were performed, but mostly after $48 \mathrm{~h}$ of positive culture. Source removal was performed within $24 \mathrm{~h}$ in $60 \%$ patients in $24-48 \mathrm{~h}$ in $15 \%$ patients and after $48 \mathrm{~h}$ in $25 \%$ patients.

\subsection{Outcome Analysis}

The all-cause crude mortality rates at 30 days and at final outcome were $37 \%(n=20)$ and $44 \%(n=24)$, respectively. A total of $15(28 \%)$ and $19(35 \%)$ patients were discharged at 30 days and at the final outcome, respectively. Interestingly, the number of patients transferred to other hospitals or discharged against medical advice was $7 \%(n=4)$ at 30 days which increased to $17 \%(n=9)$ until final outcome.

Mortality: Out of 24 deaths, 20 patients (83\%) died within 30 days of infection, while four deaths occurred after 30 days. Out of 24 deaths, 21 had bloodstream infections (88\%), 2 had respiratory tract infections (8\%) while 1 had wound infection (4\%). The 30-day crude mortality in C. auris candidemia was $44 \%$ (18 out of 41 patients). The mortality rate was higher $(53 \%)$ in patients who had a hospital stay of $<1$ month (10 out of 19 patients) than $(40 \%)$ in patients having a stay of $>1$ month (14 out of 35 patients). However, no similar effect of the duration of ICU stay was found on mortality rate. The mortality rate in patients with an ICU stay of $<1$ month was $50 \%$ (10 out of 20 patients), while it was $47 \%$ (9 out of 19 patients) in patients having an ICU stay of $>1$ month

Predictors of death: To find out any significant attributable risk factors for mortality in C. auris patients, risk factor comparison analysis was performed between two groups. Group 1-Expired patients vs. Group 2-Patients with other outcomes.

A. The prevalence of risk factors such as renal failure, congestive heart failure, haemodialysis, total parenteral nutrition and presence of devices such as invasive ventilator, central venous catheter was higher in Group1 (Expired patients) compared to Group 2 (Patients with other outcomes) (See Table 4). However, diabetes did not have any significant impact on mortality (58\% in Group-1 patients vs. $67 \%$ in Group-2 patients-OR: 0.70).

B. The mortality rate was higher in patients who either did not receive antifungal therapy $(71 \%)$ or received $<7$ days of antifungals (65\%), compared to patients who received antifungals $>7$ days (32\%). Antifungal therapy was associated with a reduction inmortality (OR-0.27).

C. Source removal was associated with lower mortality $(43 \%$ when source removal done vs. 50\% when not done-OR: 0.74 ). Likewise, source removal was associated with a higher rate of resolution of infection (38\% when source removal done vs. $21 \%$ when not done).

\subsection{Infection Control Practice Analysis}

Screening for multi-site colonization: Total 22 patients $(41 \%)$ were screened. The most commonly screened sites were axilla $(100 \%)$, groin $(82 \%)$, nares $(55 \%)$, oropharynx $(46 \%)$, rectum (41\%) and external ear canal (32\%). Out of 22 patients, $18(73 \%)$ were positive with colonization of at least one site, out of which, nine (41\%) were colonized at two or more 
sites. The positivity rates for $C$. auris among various screening specimens were as depicted in Figure 2. Nares and groin samples showed highest sensitivity.

Table 4. Analysis to determine the risk factors for mortality among C. auris cases.

\begin{tabular}{|c|c|c|c|}
\hline Risk Factor & $\begin{array}{c}\text { Group-1 } \\
\text { (Expired Patients) }\end{array}$ & $\begin{array}{c}\text { Group-2 } \\
\text { (Patients with Other Outcome) }\end{array}$ & Odds Ratio \\
\hline Renal failure & $67 \%$ & $40 \%$ & 3.0 \\
\hline $\begin{array}{c}\text { Congestive Heart } \\
\text { Failure }\end{array}$ & $46 \%$ & $17 \%$ & 4.23 \\
\hline $\begin{array}{c}\text { Invasive } \\
\text { ventilator }\end{array}$ & $75 \%$ & $63 \%$ & 1.74 \\
\hline Haemodialysis & $63 \%$ & $17 \%$ & 8.33 \\
\hline $\begin{array}{l}\text { Total parenteral } \\
\text { Nutrition }\end{array}$ & $33 \%$ & $13 \%$ & 3.25 \\
\hline $\begin{array}{c}\text { Central Venous } \\
\text { Catheter }\end{array}$ & $100 \%$ & $83 \%$ & 4.60 \\
\hline Candidemia & $88 \%$ & $67 \%$ & 3.5 \\
\hline $\begin{array}{c}\text { Bacterial } \\
\text { co-infection }\end{array}$ & $58 \%$ & $40 \%$ & 2.1 \\
\hline
\end{tabular}

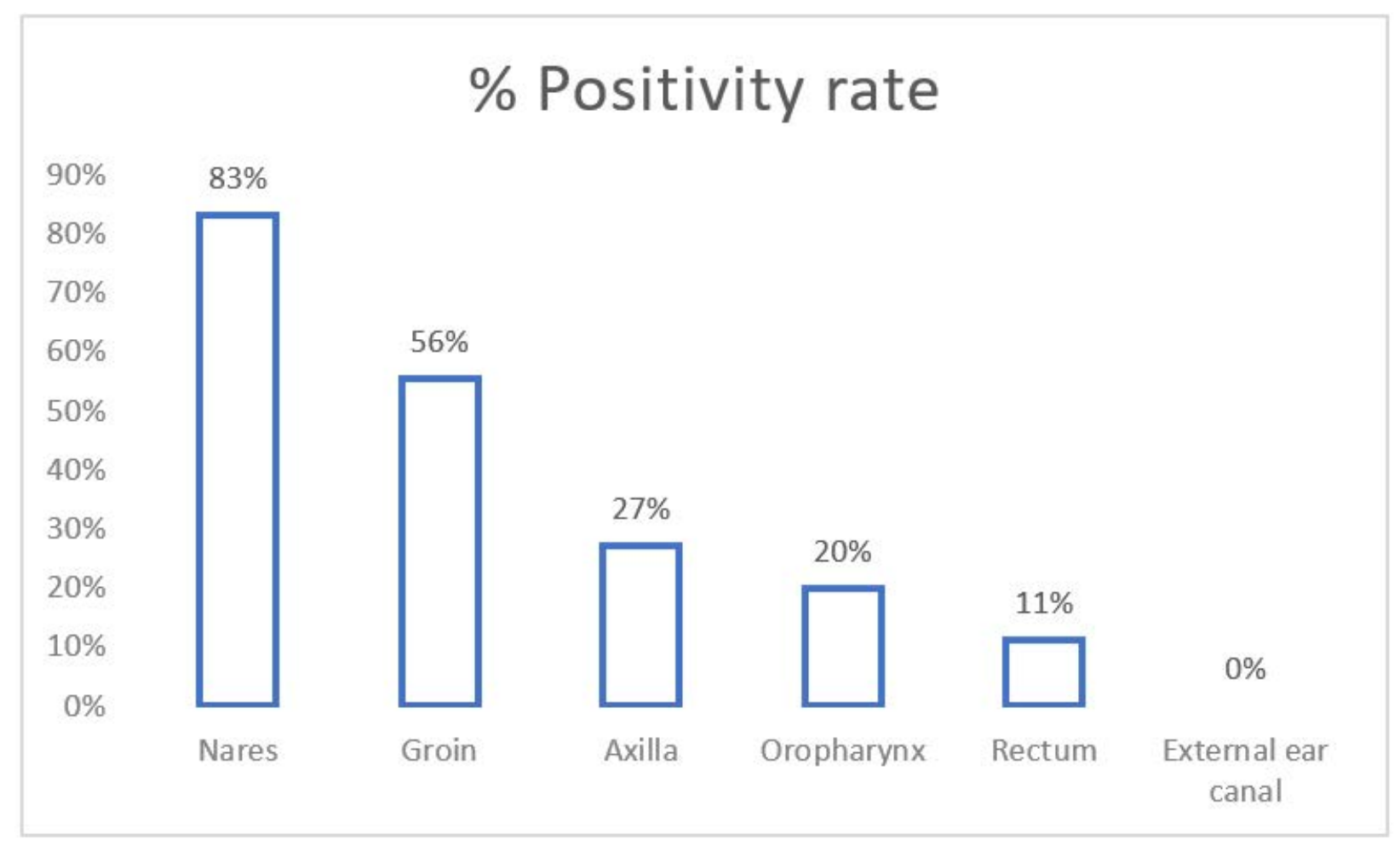

Figure 2. Positivity rate (\%) of various samples for screening of Candida auris colonization in patients.

Screening of patient contacts: The contacts of 18 patients (33\%) were screened for $C$. auris colonization. However, no contact was found to be positive.

Contact precautions for patients: Out of 54 patients, contact isolation precautions were followed in 47 patients $(87 \%)$. Out of these 47 patients, $39(83 \%)$ were placed in single isolation room, while $8(17 \%)$ were kept in the cohort.

Discontinuation of contact precautions: See Table 5 for duration of contact isolation precautions among patients. Among 47 patients placed in contact precautions, in 35 patients $(75 \%)$, contact precautions were followed until the final outcome (death-38\%, discharge- 
$36 \%)$. Other common reasons for discontinuation were microbiological clearance $(17 \%)$ and source control with treatment completion $(6 \%)$.

Table 5. Duration of contact isolation precautions.

\begin{tabular}{ccc}
\hline Days & Total & $\%$ \\
\hline$\leq 1$ week & 15 & $32 \%$ \\
1 week-1 month & 23 & $49 \%$ \\
$1-2$ month & 3 & $6 \%$ \\
$2-3$ months & 1 & $2 \%$ \\
$>3$ months & 5 & $11 \%$ \\
Grand Total & 47 & $100 \%$ \\
\hline
\end{tabular}

Surface disinfection: Out of 54 patients, in 49 patients (91\%), chlorine-based solutions with available chlorine of $>1000 \mathrm{ppm}$ were used. Phenol (5\%) was used in three patients $(6 \%)$, while no specific disinfectants solutions could be used in two patients as the patients were either expired or discharged before the availability of the reports.

\section{Discussion}

The early detection of $C$. auris infections has been shown to be beneficial, as earlier initiation of appropriate antifungal therapy saved many lives [15]. Risk factors were not different from those associated with invasive infection due to other Candida spp. [6]. The most common associated risk factors in our study were diabetes mellitus, congestive cardiac failure, chronic kidney disease, chronic pulmonary illness, presence of $\mathrm{CVC}$, urinary catheterization, post-operative drain, haemodialysis, invasive ventilation, a recent history of antibiotics and antifungal agents, chemotherapy, corticosteroid therapy, total parenteral nutrition and recent hospital/ICU stay.

Most of the reported cases of $C$. auris were isolated from blood. Other common clinical conditions include urinary tract infection, otitis, surgical wound infections, skin abscesses, peritonitis and wound infections [16,17]. In our study as well, C. auris was most commonly isolated from blood $(76 \%)$, followed by skin and soft tissue $(11 \%)$. The median time from admission to diagnosis (positive culture) was 20 days in our study, which is similar to 19 days in previous studies [18]. In our study, the order of resistance (azoles $>$ amphotericin B > echinocandins) was the same as most of the studies [6] (Table 6). However, in the present study, resistance to amphotericin B and caspofungin is significantly higher than in other studies [19]. Due to the relatively low resistance to echinocandins, it is recommended that an echinocandin empirical therapy be initiated in patients suspected of having $C$. auris infections, particularly in patients with risk factors for $C$. auris candidemia [20]. However, the drug of choice will depend on the drug susceptibility report of the isolate. In our study, $83 \%$ of patients received echinocandins, while $13 \%$ of patients received azoles as per sensitivity report; meanwhile, in a study by Arensman et al., $92 \%$ of patients received echinocandins and $8 \%$ received azoles [21]. There is currently no evidence to support combination therapy in bloodstream infections with this organism, although if the urinary tract or CNS is involved dual therapy may be necessary [22]. In our study, only one patient with Candidemia received dual antifungals: that of caspofungin and liposomal amphotericin B.

The duration of antifungal treatment appears to be similar to those used for infections caused by other Candida spp. The duration depends on microbiological clearance, clinical cureand source removal. In our study, the median duration for microbiological clearance was 20 days. Source removal approaches such as CVC removal, wound debridement has a favourable impact on reducing the antifungal duration and also in improving clinical outcomes. Thus, source removal should be done as early as possible after diagnosis. 
Table 6. Comparison table of resistance patterns for C. auris (\% of Resistant).

\begin{tabular}{ccccccc}
\hline Antifungal & Present Study & Osei et al. [19] & Lockhart et al. [23] & Chowdhary et al. [20] & Chen et al. [24] & CDC Report [11] \\
\hline Micafungin & $0 \%$ & $1.25 \%$ & $7 \%$ & $2 \%$ & $0.8 \%$ & $<\%$ \\
Anidulafungin & $5 \%$ & $1.25 \%$ & $7 \%$ & $2 \%$ & $1.1 \%$ & $<5 \%$ \\
Caspofungin & $16 \%$ & $3.48 \%$ & $7 \%$ & $2 \%$ & $12.1 \%$ & $<5 \%$ \\
Amphotericin B & $58 \%$ & $15.46 \%$ & $35 \%$ & $8 \%$ & $91 \%$ & $50 \%$ \\
Fluconazole & $78 \%$ & $44.29 \%$ & $93 \%$ & $90 \%$ & $90 \%$ \\
\hline
\end{tabular}

The all-cause 30 -day crude mortality rate was $37 \%$ in our study, which is similar to $35.2 \%$ reported by Morales-López et al. [25]. In the present study, 30-day crude mortality in C. auris candidemia was $44 \%$, which is similar to $41.9 \%$ of the study by Rudramurthy et al. [17], but lower than $61.1 \%$ of the study of Shashtri et al. [26]. Candidemia was associated with higher mortality (OR: 3.5), similar to the study of Sayeed et al. (OR-4.3). Bacterial co-infection was also associated with higher mortality (OR-2.1), similar to the study by Sayeed et al. (OR: 2.3) [6,16-22,25]. The risk factors that are significantly associated with higher mortality were congestive heart failure, renal failure, haemodialysis, invasive ventilator, CVCand total parenteral nutrition. However, diabetes was not associated with higher mortality (OR: 0.7), which is in contrast to other studies such as Sayeed et al. (OR-2.3) [16]. Considering management strategies, antifungal therapy and source removal were both associated with a significant reduction in mortality in our study. This is in line with clinical practice guidelines for treating invasive candidiasis [21].

For screening of patients for multi-site colonization of candida, CDC recommends axilla, groinand sometimes nares, while Public Health England recommends groin, axilla, urine, nose, throat, perineal swab, rectal swabor stool sample [5]. In our study, as the groin, nares and axilla have the highest positivity rate for colonization, we recommend including at least these three sites for screening. Close healthcare contacts of patients with newly identified C. auris infection or colonization should be considered for screening for $C$. auris colonization [27]. Identifying persons colonized with C. auris is a key step in containing the spread of $C$. auris. In addition, this is a useful tool for outbreak investigations. Patients on contact precautions for $C$. auris should be placed either in a single room or in cohort with patients with C. auris [28]. The CDC recommends continuing proper transmission-based precautions for the entire duration of the patient's stay in the facility. For terminal cleaning of a bedspace or room vacated by a C. auris patient, currently, hypochlorite solution is recommended at $1000 \mathrm{ppm}$ of available chlorine [29]. In a comparison of the efficacies of a range of disinfectants, sodium hypochlorite and hydrogen peroxide resulted in the greatest reduction in C. auris CFU (colony forming units) [30].

In conclusion, the present study confirms that $C$. auris is an emerging multidrugresistant yeast that presents a great challenge to global health, especially in the hospital environment. Our study has certain limitations that are inherent to a retrospective, observational study. The sample size to analyse the antifungal MIC distribution of $C$. auris isolates is not large. Furthermore, sample sizes from various centres were not big enough to allow the comparison of study objectives between centres. The study is not able to provide an attributable mortality rate due to $C$. auris infection. However, the present study confirms that $C$. auris infection carries a high risk ofmortality with candidemia in particular. C. auris isolates are largely resistant to most azoles and even to amphotericin B. Echinocandins are the most sensitive antifungals and, thus, are the first drugs of choice. Targeted anti-fungal therapy, primarily echinocandin and source control, are the most important therapeutic approaches to reduce mortality. Infection control practices such as the screening of close contacts, contact precautions and terminal cleaning are of utmost importance to identify and control the spread of $C$. auris in the hospital setup. 
Author Contributions: Conceptualization, T.F., H.C. and Y.K.G.; Data curation, N.P. (Nirav Pandya), Y.C., A.G., E.A.K. and H.E.; Formal analysis, N.P. (Nenad Pandak) and I.I.B.; Investigation, A.P. and D.P.R.; Methodology, A.U.P. and F.A. All authors have read and agreed to the published version of the manuscript.

Funding: This research received no external funding.

Institutional Review Board Statement: Ethical approval was obtained from the local ethics committee on 27 May 2019 (Approval code: 2019-39).

Informed Consent Statement: Not applicable.

Data Availability Statement: Not applicable.

Acknowledgments: Not applicable.

Conflicts of Interest: The authors declare no conflict of interest.

\section{References}

1. Wang, T.Z.; White, K.N.; Scarr, J.V.; Simon, M.S.; Calfee, D.P. Preparing your healthcare facility for the new fungus among us: An infection preventionist's guide to Candida auris. Am. J. Infect. Control 2020, 48, 825-827. [CrossRef]

2. Spivak, E.S.; Hanson, K.E. Candida auris: An emerging fungal pathogen. J. Clin. Microbiol. 2018, 56, e01588-17. [CrossRef]

3. Erdem, H.; Inan, A.; Altindis, S.; Carevic, B.; Askarian, M.; Cottle, L.; Beovic, B.; Csomos, A.; Metodiev, K.; Ahmetagic, S.; et al. Surveillance, control and management of infections in intensive care units in Southern Europe, Turkey and Iran-A prospective multicenter point prevalence study. J. Infect. 2014, 68, 131-140. [CrossRef]

4. Candida auris in Healthcare Settings-Europe. Available online: https:/ / www.ecdc.europa.eu/sites/portal/files/media/en/ publications / Publications /Candida-in-healthcare-settings_19-Dec-2016.pdf (accessed on 21 August 2020).

5. Fasciana, T.; Cortegiani, A.; Ippolito, M.; Giarratano, A.; Di Quattro, O.; Lipari, D.; Graceffa, D.; Giammanco, A. Candida auris: An overview of how to screen, detect, test and control this emerging pathogen. Antibiotics 2020, 9, 778. [CrossRef] [PubMed]

6. Sarma, S.; Upadhyay, S. Current perspective on emergence, diagnosis and drug resistance in Candida auris. Infect. Drug Resist. 2017, 10, 155-165. [CrossRef] [PubMed]

7. Council of State \& Territorial Epidemiologists (CSTE) Position Statement(s) 18-ID-05. Candida auris 2019 Case Definition. CDC Website. Available online: https://wwwn.cdc.gov/nndss/conditions/candida-auris/case-definition/2019/ (accessed on 31 August 2020).

8. Kordalewska, M.; Perlin, D.S. Identification of drug resistant Candida auris. Front. Microbiol. 2019, 10, 1918. [CrossRef] [PubMed]

9. Ostrowsky, B.; Greenko, J.; Adams, E.; Quinn, M.; O’Brien, B.; Chaturvedi, V.; Berkow, E.; Vallabhaneni, S.; Forsberg, K.; Chaturvedi, S.; et al. Candida auris isolates resistant to three classes of antifungal medications-New York, 2019. MMWR. Morb. Mortal. Wkly. Rep. 2020, 69, 6-9. [CrossRef]

10. Recommendations for Identification of Candida auris. Available online: https://www.cdc.gov/fungal/diseases/candidiasis / recommendations.html (accessed on 12 January 2021).

11. Information for Laboratorians and Health Professionals. Available online: https://www.cdc.gov/fungal/candida-auris/healthprofessionals.html (accessed on 12 January 2021).

12. Arendrup, M.C.; Prakash, A.; Metetiadis, J.; Sharma, C.; Chowdhary, A. Comparison of EUCAST and CLSI reference microdilution MICs of eight and associated tentative. Antimicrob. Agents Chemother. 2017, 61, e00485-17. [CrossRef]

13. Candida auris Antifungal Susceptibility Testing. Available online: https://www.cdc.gov/fungal/candida-auris/c-aurisantifungal.html (accessed on 12 January 2021).

14. Candida auris, Treatment and Management of Infections and Colonization. Available online: https://www.cdc.gov/fungal/ candida-auris / c-auris-treatment.html (accessed on 12 January 2021).

15. Chowdhary, A.; Voss, A.; Meis, J.F. Multidrug-resistant Candida auris: 'New kid on the block' in hospital-associated infections? J. Hosp. Infect. 2016, 94, 209-212. [CrossRef]

16. Sayeed, M.A.; Farooqi, J.; Jabeen, K.; Awan, S.; Mahmood, S.F. Clinical spectrum and factors impacting outcome of Candida auris: A single center study from Pakistan. BMC Infect. Dis. 2019, 19, 384. [CrossRef]

17. Rudramurthy, S.M.; Chakrabarti, A.; Paul, R.A.; Sood, P.; Kaur, H.; Capoor, M.R.; Kindo, A.J.; Marak, R.S.K.; Arora, A.; Sardana, R.; et al. Candida auris candidaemia in Indian ICUs: Analysis of risk factors. J. Antimicrob. Chemother. 2017, 72, 1794-1801. [CrossRef] [PubMed]

18. Mizusawa, M.; Miller, H.; Green, R.; Lee, R.; Durante, M.; Perkins, R.; Hewitt, C.; Simner, P.J.; Carroll, K.C.; Hayden, R.T.; et al. Can multidrug-resistant Candida auris be reliably identified in clinical microbiology laboratories? J. Clin. Microbiol. 2017, 55, 638-640. [CrossRef] [PubMed]

19. Osei Sekyere, J. Candida auris: A systematic review and meta-analysis of current updates on an emerging multidrug-resistant pathogen. MicrobiologyOpen 2018, 7, 1-29. [CrossRef] [PubMed] 
20. Chowdhary, A.; Prakash, A.; Sharma, C.; Kordalewska, M.; Kumar, A.; Sarma, S.; Tarai, B.; Singh, A.; Upadhyaya, G.; Upadhyay, S.; et al. A multicentre study of antifungal susceptibility patterns among 350 Candida auris isolates (2009-17) in India: Role of the ERG11 and FKS1 genes in azole and echinocandin resistance. J. Antimicrob. Chemother. 2018, 73, 891-899. [CrossRef] [PubMed]

21. Arensman, K.; Miller, J.L.; Chiang, A.; Mai, N.; Levato, J.; LaChance, E.; Anderson, M.; Beganovic, M.; Dela Pena, J. Clinical outcomes of patients treated for Candida auris infections in a multisite health system, Illinois, USA. Emerg. Infect. Dis. 2020, 26, 866-871. [CrossRef]

22. Griffith, N.; Danziger, L. Candida auris urinary tract infections and possible treatment. Antibiotics 2020, 9, 898. [CrossRef]

23. Lockhart, S.R.; Etienne, K.A.; Vallabhaneni, S.; Farooqi, J.; Chowdhary, A.; Govender, N.P.; Colombo, A.L.; Calvo, B.; Cuomo, C.A.; Desjardins, C.A.; et al. Simultaneous emergence of multidrug-resistant Candida auris on 3 continents confirmed by whole-genome sequencing and epidemiological analyses. Clin. Infect. Dis. 2017, 64, 134-140. [CrossRef]

24. Chen, J.; Tian, S.; Han, X.; Chu, Y.; Wang, Q.; Zhou, B.; Shang, H. Is the superbug fungus really so scary? A systematic review and meta-analysis of global epidemiology and mortality of Candida auris. BMC Infect. Dis. 2020, 20, 827. [CrossRef]

25. Morales-López, S.E.; Parra-Giraldo, C.M.; Ceballos-Garzón, A.; Martínez, H.P.; Rodríguez, G.J.; Álvarez-Moreno, C.A.; Rodríguez, J.Y. Invasive infections with multidrug-resistant yeast Candida auris, Colombia. Emerg. Infect. Dis. 2017, 23, 162-164. [CrossRef]

26. Shastri, P.S.; Shankarnarayan, S.A.; Oberoi, J.; Rudramurthy, S.M.; Wattal, C.; Chakrabarti, A. Candida auris candidemia in an intensive care unit-Prospective observational study to evaluate epidemiology, risk factors, and outcome. J. Crit. Care 2020, 57, 42-48. [CrossRef]

27. Pappas, P.G.; Kauffman, C.A.; Andes, D.R.; Clancy, C.J.; Marr, K.A.; Ostrosky-Zeichner, L.; Reboli, A.C.; Schuster, M.G.; Vazquez, J.A.; Walsh, T.J.; et al. Clinical practice guideline for the management of candidiasis: 2016 update by the Infectious Diseases Society of America. Clin. Infect. Dis. 2016, 62, e1-e50. [CrossRef]

28. Ku, T.S.N.; Walraven, C.J.; Lee, S.A. Candida auris: Disinfectants and implications for infection control. Front. Microbiol. 2018, 9 , 726. [CrossRef] [PubMed]

29. Kean, R.; Sherry, L.; Townsend, E.; McKloud, E.; Short, B.; Akinbobola, A.; Mackay, W.G.; Williams, C.; Jones, B.L.; Ramage, G. Surface disinfection challenges for Candida auris: An in-vitro study. J. Hosp. Infect. 2018, 98, 433-436. [CrossRef] [PubMed]

30. Ledwoch, K.; Maillard, J.Y. Candida auris dry surface biofilm (DSB) for disinfectant efficacy testing. Materials 2018, 12, 18. [CrossRef] [PubMed] 